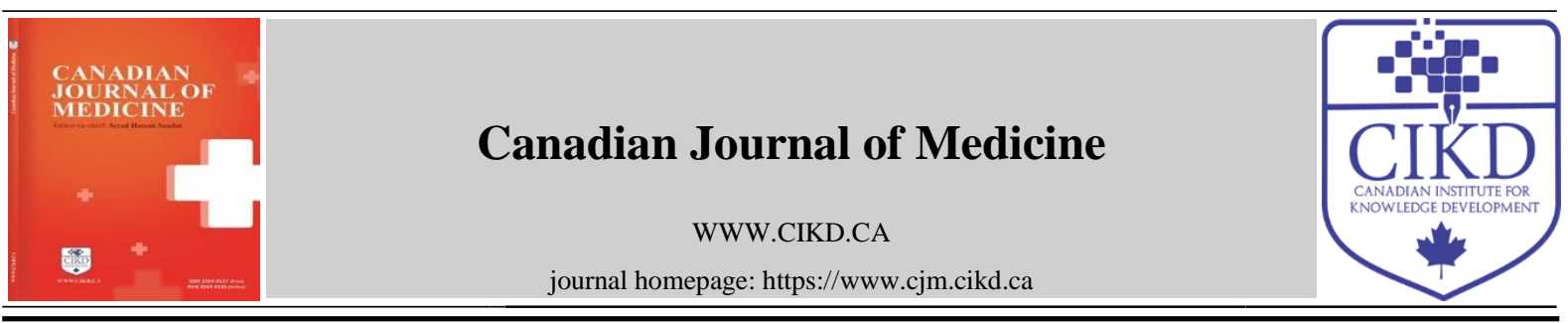

\title{
Hyperbaric Oxygen Therapy for Carbon Monoxide Poisoning can Lead a Perfect Result: A Case Report
}

\author{
Canan Bolcu Emir ${ }^{1 *}$, Selin Gamze Sümen², Tuna Gümüşs ${ }^{3}$ \\ ${ }^{1}$ SBU Okmeydani Education and Training Hospital, Istanbul, Turkey \\ ${ }^{2}$ University of Health Sciences, Hamidiye Medical Faculty, Department of Underwater and \\ Hyperbaric Medicine, Istanbul, Turkey \\ ${ }^{3}$ University of Health Sciences, Kartal Dr Lütfi Kirdar City Hospital, Department of Underwater \\ and Hyperbaric Medicine, Istanbul, Turkey
}

\begin{abstract}
Keywords:

Carbon monoxide poisoning, Neurological complications, Hyperbaric oxygen therapy

\section{Received}

26 June 2021

Received in revised form

07 July 2021

Accepted

Carbon monoxide poisoning (COP) is a common emergency worldwide, especially in the wintertime. It is known to cause serious morbidities, resulting in high mortality risk. COP is also reported to result in neurological complications for which hyperbaric oxygen therapy is likely to offer some benefits, which certainly makes it one of the potential treatment modalities. Here, Case in the paper is of a 64-year-old female patient with carbon monoxide poisoning. Her neurological symptoms nearly disappeared after the application of hyperbaric oxygen therapy (HBOT) and conventional medical treatment. Our case guides for the key role of HBOT in the treatment process of a 64-year-old female patient with COP. Our clinical experience revealed that hyperbaric oxygen therapy could successfully reverse the symptoms of delayed neurological findings caused by COP, a serious and preventable health problem.
\end{abstract}

09 July 2021

*Correspondence:

emir.canan@yahoo.com.tr

\section{Introduction}

Carbon monoxide poisoning is one of the most encountered emergencies worldwide related to toxic gases, stemming from inadequate combustion of carbon fuels which results in a lifethreatening health problem. Furthermore, it causes serious morbidities, exerting negative 
impacts on the quality of life of the patient who presents symptoms of delayed neurological sequelae. Acute poisoning is usually treated with administration of normobaric oxygen treatment (NBOT), improvement of hemodynamic status, and application of additional medical treatments determined by the general health status of the patient [1]. Due to the fact that central nerve system is primarily affected in severely intoxicated cases, patient might be admitted to the hospital with the symptoms suggestive of impairment of higher cerebral functions. Moreover, there is no definitive consensus regarding the diagnosis and treatment management of the delayed sequelae of carbon monoxide intoxication. Development of delayed neuropsychiatric sequelae, including dementia, psychosis, parkinsonism, chorea and incontinence are the long-term consequences of $\mathrm{CO}$ poisoning, which result in some concerns [2]. Hence, delayed neurologic sequelae of COP should be recognized as early as possible by other clinicians such as neurologists and psychiatrists. Although response to different treatment modalities varies from patient to patient, but hyperbaric oxygen treatment should be taken into consideration as a treatment option for patients.

In this case report, we aimed to report a patient who was initially misdiagnosed with dementia but should have been diagnosed with neurological late sequelae of COP, instead. Her successful recovery with HBOT was discussed in this report, as well.

\section{Case Presentation}

A 64-years-old female patient was referred to neurology policlinic with pre-diagnosis of dementia. Her relatives have been complaining about her unresponsiveness, slow motions and apathy in the last month. While the patient had being questioned regarding not only the symptoms but also the duration of complaints, they mentioned about her exposure to carbon monoxide poisoning caused by a stove in the previous month. The patient was found unconscious early in the morning by her relatives and she was primarily admitted to the emergency department at the regional district hospital and was then treated with the first-line therapies. Subsequently, she was referred to neurology department for her comprehensive neurological examination due to the persistent neurological symptoms. On her neurological examination, she was conscious, but her cooperation was poor as she gave only very short answers slowly. Besides, her gestures slowed down as a consequence of the rigidity on both her arms and legs causing her walk with small steps, which was only possible with bilateral assistance. In conclusion, she had some symptoms of Parkinson disease with poor cognition.

After taking her blood samples, the results of hematological markers, biochemistry tests were all in normal range. Then, we planned to perform alertness electroencephalography (EEG), magnetic resonance imaging of the brain and mini-mental state examination test. She was found to have diffused high signal intensity involving subcortical white matter, globus pallidus on FLAIR and T2W images of the cranial magnetic resonance imaging (MRI). On the contrary, no abnormal contrast enhancement was observed in the above areas (Figure 1a and 1b). Her routine EEG test during awareness exhibited generalized slow waves with delta- and theta-wave activity (Figure 2). 


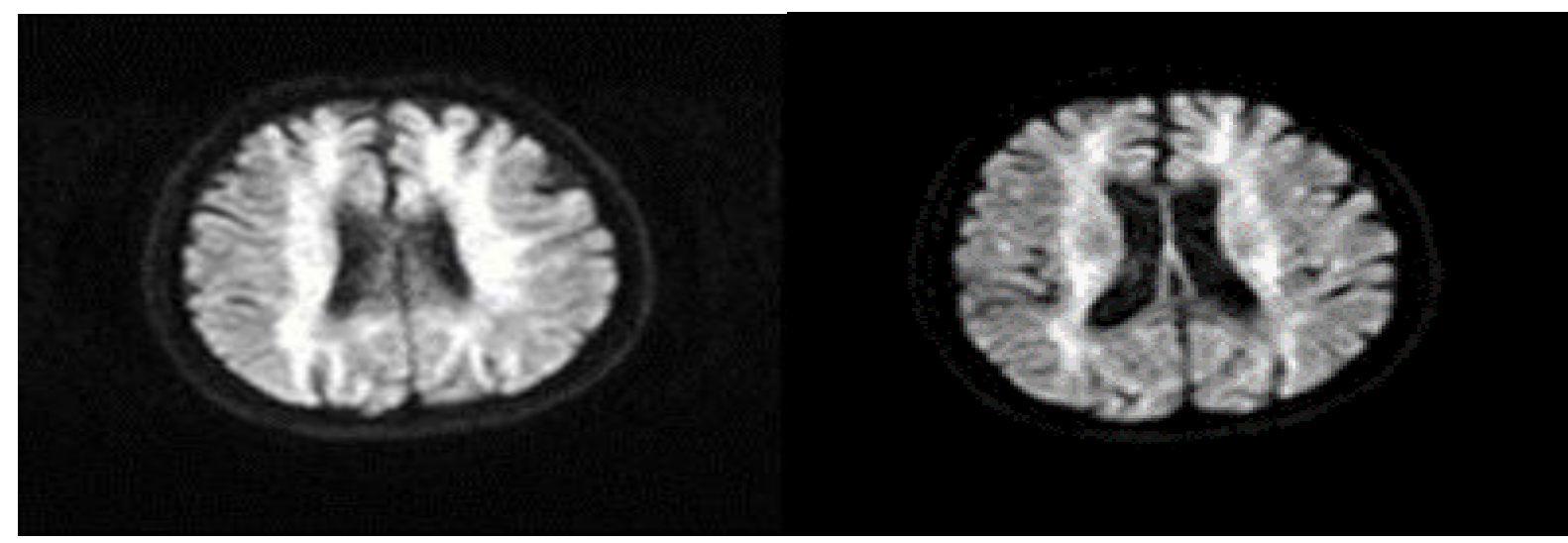

(a)

(b)

Figure 1. Pretreatment T2W brain MRI, showing bilateral diffuse subcortical hyperintensity

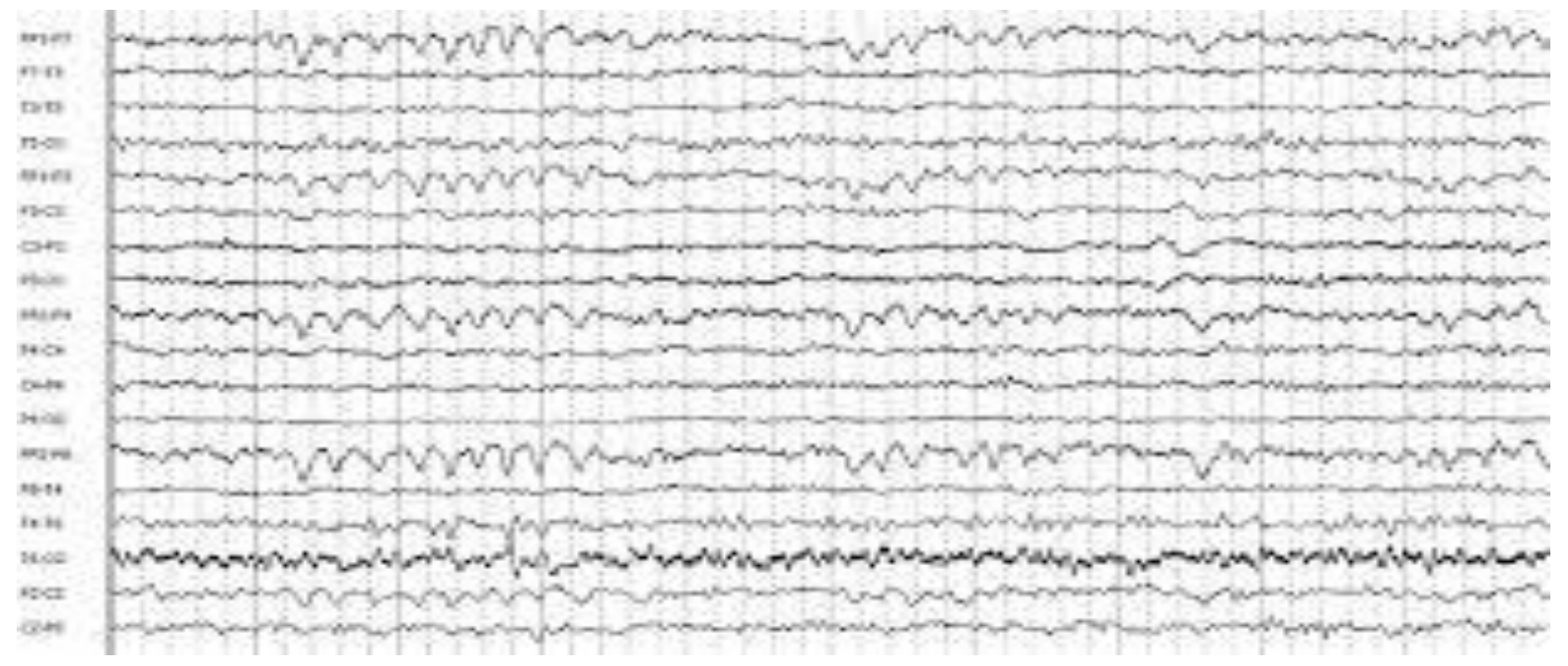

Figure 2. EEG showed generalized theta- and delta-wave activity

Due to low level of patient's cooperation, it could not be possible to take the mini-mental state examination test. After neurological evaluation, the patient was prescribed memantine 20 $\mathrm{mg} /$ day and levodopa $250 \mathrm{mg} /$ day so as to regress the complaints. Moreover, the clinical diagnosis was reevaluated by taking into consideration of several factors such as previous history of $\mathrm{CO}$ intoxication, neurological examination findings, the revealing of brain MRI and EEG; they all led us the diagnosis of delayed neuropsychiatric sequelae of previous CO poisoning. Then, she was referred to the department of hyperbaric oxygen treatment for the consultation of an underwater and hyperbaric physician. They concluded that the patient had been suffering from delayed sequelae of COP and they decided to administer HBOT. Videotapes of the patient were also recorded with the purpose of comparing the physical examination findings regarding pre-and post-treatments . The patient was treated with $100 \%$ oxygen at 2.4 ATA, for 120 minutes in a multi-place chamber. After 30 treatment sessions, an overall healing of the symptoms was observed. Apart from such beneficial effects of the treatment, the patient quite well tolerated hyperbaric oxygen treatment process without any signs of side effects. After the completion of HBOT, she was admitted to our neurology policlinic again one month later. She was smiling, her speech was more fluent, she was able to walk without assistance. We learnt that she was able to do her routine daily activities by herself 
at home. We gradually stopped levodopa treatment and decreased memantine dose to $10 \mathrm{mg}$ daily. Her MRI results subsequent to the completion of HBO therapy showed a regression in the lesions on T2W images when compared to the pre-treatment period (Figure 3a and 3b).

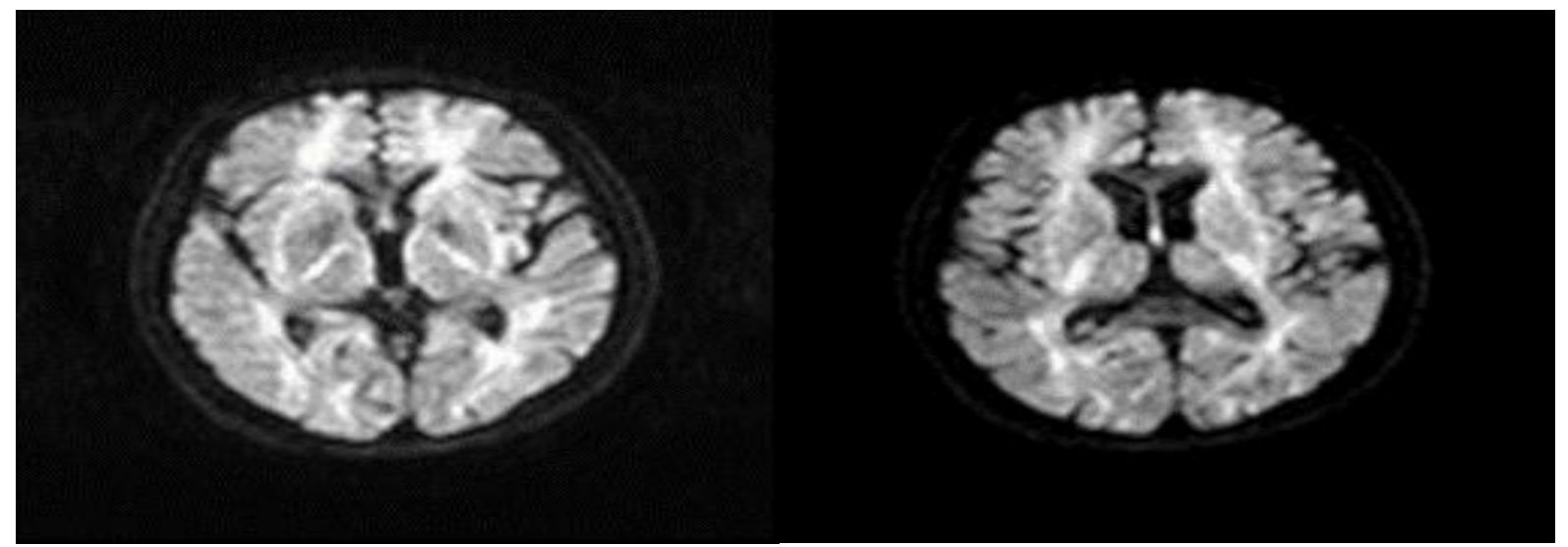

(a)

(b)

Figure 3. Post-treatment T2 W MRI showed regression in the lesions

We reported a patient who was misdiagnosed as dementia with the symptoms of delayed neurologic sequelae of COP and she successfully recovered with HBOT supported with medical treatment. We discussed the clinical picture, follow up period and her treatment with the literature.

\section{Discussion}

As it is clearly defined, $\mathrm{CO}$ is an odorless, colorless and highly toxic gas for all cells resulting from incomplete combustion of fuels such as coal and wood. When this toxic gas is inhaled accidently, it has deleterious effects primarily on nervous system cells and disrupts cellular functions of several organs. Poisoning is often caused by the inhalation of $\mathrm{CO}$ accumulated in the ambient air of a closed environment as a consequence of use of different sources such as fire, stoves, barbecues, water heaters, and vehicle or compressor exhaust [1, 2]. CO poisoning, which is frequently encountered in winter, may result in sudden deaths due to the fact that it is not diagnosed and intervened promptly as a result of which most deaths occur during sleep at night, in particular. The results of the retrospective study published by Can et al are quite striking. CO-related death risk in our country has been determined as 0.35/100.000. It has been also reported that the incidents have occurred mostly in winter, $78 \%$ of which has stemmed from the toxic gas from stoves and majority of these victims have been reported to be male [3].

It is a well-known fact that there is a "competition" between oxygen and $\mathrm{CO}$ in the provision of oxygen for the tissues. The accumulation of carbon monoxide in tissues, in this regard, may cause the very same tissues suffering from oxygen deprivation. In other words, inhaled CO quickly binds to red blood cells in the blood, preventing oxygen from being transported to the cells. As a result, cells, tissues and organs cannot provide the oxygen required for vital functions. Hence, hypoxia is considered to be responsible for the pathophysiology of the disease. This is because, the lack of oxygen required for the cell to survive causes some temporary or permanent damage to the tissues and organs in the body. Primarily, the brain, heart and circulatory system, as well as respiratory system, kidneys, and liver are more susceptible to oxygen deficiency and they are promptly affected. As a consequence, symptoms 
concerning the dysfunction or malfunction of affected organs may appear commonly following intoxication caused by carbon monoxide.

Clinical signs and symptoms vary due to risk factors such as age, duration of exposure, source of CO, presence of additional disease, delay in diagnosis and treatment. The exposure may result in serious clinical signs in the elderly, children and pregnant women. Furthermore, it is classified in three groups as mild, moderate and severe. Poisoning may have mild symptoms such as nausea, vomiting, abdominal pain, dizziness, sweating, and fatigue, as well as signs related to the toxic effect of nervous system such as loss of consciousness, fainting, visual impairment, memory impairment, and hearing loss.

Scientific studies have shown that $\mathrm{CO}$ affects especially the nervous system and brain in the late period. Delayed neurological findings can appear within 3-240 days after recovery in approximately $40 \%$ of cases of severe CO poisoning. Patients usually present symptoms pointing delayed sequelae such as cognitive impairments in varying degrees, personality changes, movement disorders, incontinence, gait, speech impairment, involuntary movements, and memory deficits $[4,5,6]$.

In delayed neurological findings associated with $\mathrm{CO}$ intoxication, the main pathological feature is the reversible demyelinating process in cerebral white matter [5]. Chang et al. reviewed magnetic resonance images obtained from 15 patients with delayed neuropsychiatric symptoms after acute carbon monoxide intoxication. Long repeat images showed periventricular white matter and central semi-precious bilaterally symmetric combined high signal intensity in all patients. They also demonstrated presence of bilateral diffuse low intensity signal in the thalamus and putamen in 10 patients. Nine patients had bilateral ischemia or globus pallidus necrosis [5].

In CO poisoning, treatment begins at the scene. Survivors who are found accidentally in environments contaminated with $\mathrm{CO}$ leakage from different sources should be removed from the scene promptly with the suspicion of poisoning. Inhaling $100 \%$ oxygen at the highest concentrations, normobaric oxygen therapy, is the vital approach to remove toxic $\mathrm{CO}$ from all tissues rapidly. Normobaric oxygen therapy can be applied with 10-15 liters/minute free oxygen flow rate with non-reversible masks with reservoir bag that can cover mouth and nose. Despite the administration of high flow rate oxygen, some cases may still require intensive and high concentration of oxygen. For this purpose, hyperbaric oxygen therapy is preferred to be administered in the treatment process of moderate and severe cases of $\mathrm{CO}$ intoxication showing the following signs or symptoms: coma, transient loss of consciousness, convulsion, ischemic heart disease, neurological complaints, end organ damage, pregnancy, worsening of the symptoms despite normo-baric oxygen treatment, severe metabolic acidosis, and a carboxyhemoglobin (COHb) level above $25 \%$ [7, 8, 9, 10].

Hyperbaric oxygen therapy is the treatment which is administered by breathing $100 \%$ oxygen intermittently to the patient at a pressure above one atmosphere absolute (1ATA $=760$ $\mathrm{mmHg}$ ) in a closed environment. Patients usually inhale oxygen by means of mask, endotracheal tube or a hood.

Hyperbaric oxygen treatment increases level of oxygen dissolved in the plasma of patients and the amount of oxygen delivered to the tissues. In addition, oxygen competes with carbon monoxide bound to red blood cells and tissues as mentioned previously. $\mathrm{CO}$ is rapidly excreted from the body by means of high concentration of oxygen. Thus, the complaints begin to regress 
rapidly, depending on the speed of excretion from the body. Furthermore, it is commonly known that late neurological symptoms, which emerge in weeks and months after the first treatment, may be effectively treated with HBOT [10].

HBOT plays an active role in the treatment of $\mathrm{CO}$ poisoning, especially exerting antihypoxic and antiedema effects. During HBO administration, hypoxia is eliminated by dissolving high levels of oxygen in body fluids and tissues, especially in blood. In addition, oxygen results in vasoconstriction which regulates interstitial edema and improves tissue perfusion [11].

In a randomized double-blind study, Weaver et al. has showed that the incidence of delayed neurological sequelae due to COP in the early and late period (6 weeks - 6 months - 12 months) decreased significantly in patients treated by hyperbaric oxygen. The very same study has also suggested that delayed neurological sequelae could be prevented in one out of every six patients who have been provided with HBOT [9].

In a meta-analysis study conducted by Lin et al., it has been demonstrated that patients administered HBOT in CO poisoning had a lower incidence of neuropsychological sequelae in terms of headache, memory impairment, concentration difficulties, sleep disturbance, and delayed neurological sequelae compared to those treated with normobaric oxygen [8].

In the study of Sar1 Doğan et al., it is stated that the presence of neurological findings at the admission to the hospital is significant in terms of predicting the risk of delayed neurological sequelae. Therefore, it is necessary to inform patients and their relatives comprehensively about delayed neuropsychiatric complaints at discharge. It is not correlated in terms of the $\mathrm{COHb}$ level and the severity of clinical symptoms, but the $\mathrm{COHb}$ level makes a definitive diagnosis. In the study, no statistically significant relationship was found between $\mathrm{COHb}$ levels and delayed neurological sequelae [12].

Preventing of misdiagnosis in $\mathrm{CO}$ poisoning requires a high level of suspicion. In this case report, we presented the results of hyperbaric oxygen therapy in a 64-year-old patient who had been initially misdiagnosed with dementia. We have been able to improve the quality of life of the patient with the administration of HBOT. Hyperbaric oxygen treatment might be a cornerstone of multifaceted approach to treatment in order to provide better outcome of prognosis with CO poisoned patients. Development of delayed neuropsychiatric sequelae, including dementia, psychosis, parkinsonism, chorea and incontinence are the feared long-term consequences of COP. Thus, delayed neurologic sequelae of COP should be well recognized by other clinicians such as neurologists and psychiatrists [13]. Although response to different treatment modalities varies in individuals, patient selection for HBOT should be done carefully [14]. Carbon monoxide poisoning, is not only a life-threatening health problem, but also it may exert deleterious effects on the quality of life of the patient with its delayed sequelae.

\section{Conclusions}

In conclusion, it was revealed in our clinical experience that hyperbaric oxygen therapy could successfully reverse the symptoms of delayed neurological findings caused by COP which is one of the serious and preventable health problems. Due to the delayed neurological findings of the disease, patients should be consulted with the department of hyperbaric oxygen treatment to provide better results and to improve the prognosis. Apart from this, hyperbaric oxygen treatment helps to alleviate the economic burden on society by increasing survival. 


\section{Conflict of interest}

\section{There is no conflict of interest.}

\section{Founding}

No subsidies or grants has contributed to the work.

\section{References}

[1] Walker E. Carbon monoxide poisoning. BMJ. 1999;23: 1082-1083.

[2] Iqbal S, Clower JH, Saha S, et al. Residential carbon monoxide alarm prevalence and ordinance awareness. Journal of Public Health Management and Practice JPHMP. 2012;18(3):272-278.

[3] Can G., Sayılı U., Aksu Sayman Ö. et al. Mapping of carbon monoxide related death risk in Turkey: a ten-year analysis based on news agency records. BMC Public Health. 2019; 3:19(1):9.

[4] Mazo J, Mukhtar E, Mazo Y, Nagaraj A, Mantello MT. Delayed brain injury post carbon monoxide poisoning. Radiol Case Rep. 2020;10-15(10):1845-1848.

[5] Chang KH., Han MH., Kim HS., Wie BA., Han MC. Delayed encephalopathy after acute carbon monoxide intoxication: MR imaging features and distribution of cerebral white matter lesions. Radiology. 1992; 184(1):117-122.

[6] Pepe G, Castelli M, Nazerian P. et al. Delayed neuropsychological sequelae after carbon monoxide poisoning: predictive risk factors in the Emergency Department. A retrospective study. Scand J Trauma Resusc Emerg Med. 2011;17(19):16.

[7] Kenneth P. Stoller (2013) Hyperbaric oxygen and carbon monoxide poisoning: a critical review. Neurological Research. 2013;29(2):146-155.

[8] Lin CH, Su WH, Chen YC, Feng PH, Shen WC, Ong JR, Wu MY, Wong CS. Treatment with normobaric or hyperbaric oxygen and its effect on neuropsychometric dysfunction after carbon monoxide poisoning: A systematic review and meta-analysis of randomized controlled trials. Medicine (Baltimore). 2018; 97(39):e12456.

[9] Weaver LK, Hopkins RO, Chan KJ, Churchill S, Elliott CG, Clemmer TP, Orme JF Jr, Thomas FO, Morris AH . Hyperbaric oxygen for acute carbon monoxide poisoning. N Engl J Med. 2002;347(14):1057-67.

[10] Weaver LK, Valentine KJ, Hopkins RO. Carbon monoxide poisoning: risk factors for cognitive sequelae and the role of hyperbaric oxygen. Am J Respir Crit Care Med. 2007;176(5):491-7.

[11] Hampson NB, Weaver LK. Carbon monoxide poisoning: a new incidence for an old disease. Undersea Hyperb Med. 2007; 34(3):163-8.

[12] Sarı Doğan F, Güneysel Ö, Gökdağ E, Güneş M, Sümen SG . Demographic characteristics and delayed neurological sequelae risk factors in carbon monoxide poisoning. Am J Emerg Med. 2020;38(12):2552-2556.

[13] Prockop LD, Chichkova R I. Carbon monoxide intoxication: An updated review. Journal of the Neurological Sciences. 2007;262:122-130.

[14] Pappalardo I, Lorenzo C, Arena L, Testa L, Giovanniello A, Cava M, et al. Is "delayed" hyperbaric therapy effective for "delayed" encephalopathy after carbon monoxide intoxication? eNeurologicalSci. 2019;5:18:100219. 